Case Report

\title{
Intestinal Phytobezoar: A Rare Case Report
}

\author{
Ahmed $\mathrm{M}^{\mathrm{a}}$, Afroze $\mathrm{N}^{\mathrm{b}}$, Rahman AJENc , Sabiha $\mathrm{M}^{\mathrm{d}}$
}

\begin{abstract}
A bezoar is the result of the ingestion of indigestible or poorly digestible substances that accumulate in the gastrointestinal tract in the form of a mass. Among different types of bezoars phytobezoars is the most common type and formed by excessive consumption of herbal nutrients containing high amounts of indigestible fibers. There are some predisposing factors for gastrointestinal phytobezoars formation such as loss of pyloric function, decreased gastric motility, malabsorption, inadequate chewing and excessive consumption of herbal indigestible fibers. Small bowel obstruction is the most common major complication of phytobezoars. We present a rare case of intestinal obstruction in a middle aged man who was previously diagnosed to have malabsorption and used to take herbal medicine. He was treated successfully by right hemicolectomy under general anesthesia. On histopathological examination a large phytobezoar was found in the terminal part of ileum.
\end{abstract}

Key words: Intestinal phytobezoar, Case Report

(BIRDEM Med J 2017; 7(1): 64-67)

\section{Introduction}

Bezoar is defined as the accumulation of undigested foreign bodies or nutrients in gastrointestinal tract. ${ }^{1}$ Depending on the content, there are phytobezoars that are composed of plant material fibers, trichobezoars that are composed of hair, lactobezoars that are formed in infants from constant intake of inspissated milk, pharmacobezoars that are formed by sustained release medications, etc. ${ }^{2}$ Among these phytobezoars are the most common. ${ }^{3}$ Abdominal distress, abdominal pain, constipation, nausea and vomiting are common presentations. ${ }^{4}$ Complications are intestinal obstruction, perforation, ulcer and melena. ${ }^{5}$ Among these small bowel obstruction is the most common major complication of phytobezoars and accounts for 0.4$4 \%$ of total cases of intestinal obstruction. ${ }^{6-7}$

\section{Author Informations}

a. Dr. Mousumi Ahmed, Associate Professor, Department of Pathology, BIRDEM General, Hospital, Dhaka

b. Dr. Nazma Afroze, Professor \& Head, Department of Pathology, BIRDEM General Hospital, Dhaka

c. Dr. AJE Nahar Rahman, Honorary Professor of Pathology, BIRDEM General Hospital, Dhaka

d. Dr. Mahjabin Sabiha, Medical Officer, Department of Pathology, BIRDEM General Hospital, Dhaka

Address of Correspondence: Mousumi Ahmed, Associate Professor, Department of Pathology, BIRDEM, Hospital, Dhaka. E-mail: mousumi7406@gmail.com

Received: January 12, 2016

Accepted: November 30, 2046
Plain abdominal radiography in the standing position, abdominal ultrasound and abdominal computed tomography are used for the diagnosis of intestinal phytobezoars. $^{8}$

The methods of removal of bezoars depends on the locations as well as sizes of the bezoars and on associated complications. ${ }^{1}$ Gastric lavage and endoscopic or surgical removal are used in cases of gastric phytobezoars. If bezoars are located in terminal region of the colon, conservative treatment with enemas or resins are first given and if conservative treatment fails, or in complicated cases surgical treatment either by open or laparoscopic technique are done. Main surgical techniques include manual fragmentation and milking into caecum, enterotomy, as well as resection and anastomosis in complicated cases. ${ }^{3}$

We present a case of small intestinal phytobezoar in a middle aged man who had a history of malabsorption for nine years and used to take herbal medicine. He was treated successfully by right hemicolectomy under general anesthesia. In this paper we report our experience with the case, along with a literature review.

\section{Case Report}

A 48 year-old male with middle class background was admitted in the Surgery Department of BIRDEM General Hospital with the complaints of abdominal pain as well as distention and absence of defecation for the 
last three days. He was a diagnosed case of malabsorption for the last nine years and underwent for duodenal biopsy in the GHPD unit of the same hospital nine years back. Histopathology report of the biopsy sample revealed chronic duodenitis with partial villous atrophy. He was given proper treatment for malabsorption at that time and was advised for regular follow up. The patient also began to take herbal medicine from a kabiraj for the last few years.

During admission his vital parameters were normal and his general physical examination results were unremarkable. Abdominal examination revealed distention, tenderness and an irregular mass in the right lower quadrant of the abdomen.

The results of routine haematological and biochemical laboratory studies revealed only anaemia with a hemoglobin level of $12.0 \mathrm{gm} / \mathrm{dL}$. Abdominal ultrasonography revealed excessive bowl gas and a $7 x 6 \mathrm{~cm}$ mass at ileocaecal junction.

Along with these results, the diagnosis of intestinal obstruction due to iliocaecal mass was made. Under general anesthesia right sided hemicolectomy was done and the specimen was sent to the Department of Histopathology and Cytopathology of the same institute for further evaluation. Macroscopic examination of the $85 \mathrm{~cm}$ long right hemicolectomy specimen revealed a $9 \mathrm{x}$ $5 \times 5 \mathrm{~cm}$ circular ball of undigested food fibers obstructing the ileal lumen at ileocaecal junction. The ileum proximal

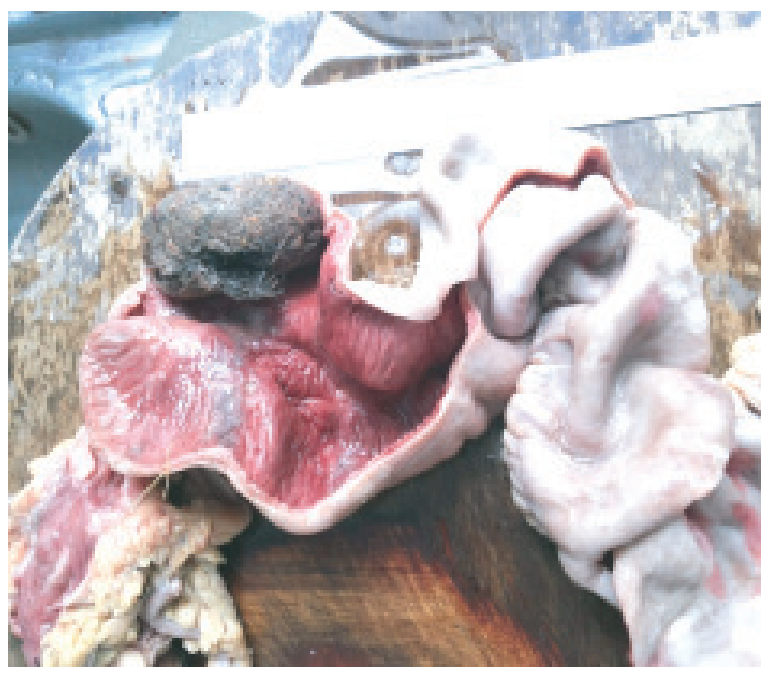

Figure 1: Macroscopic appearance of the right hemicolectomy specimen. The ileum was dilated, congested and flat (thin arrow). The lumen showed a ball of undigested food fibers (thick arrow) to the obstruction was dilated. Throughout the whole length the ileal mucosa was also congested, flat and devoid of mucosal folds. (Fig.-1) Other parts of the specimen were unremarkable. Microscopic examination of the ball showed undigested food material and vegetable cells. (Fig.-2) Ileum showed evidence of ileitis and atrophied villi. With the above macroscopic and microscopic findings a diagnosis of phytobezoar with ileitis and villous atrophy was made. (Fig.-3)

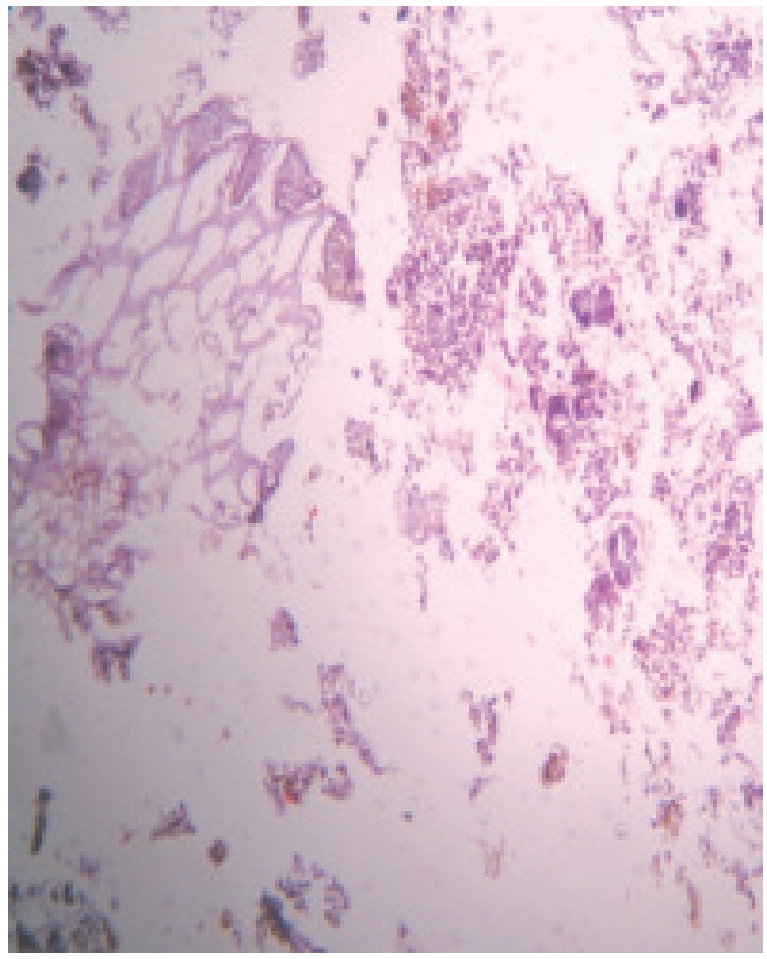

Figure 2: Microscopic picture of ball of undigested food fibers. Arrow indicating vegetable fiber. (10x10)

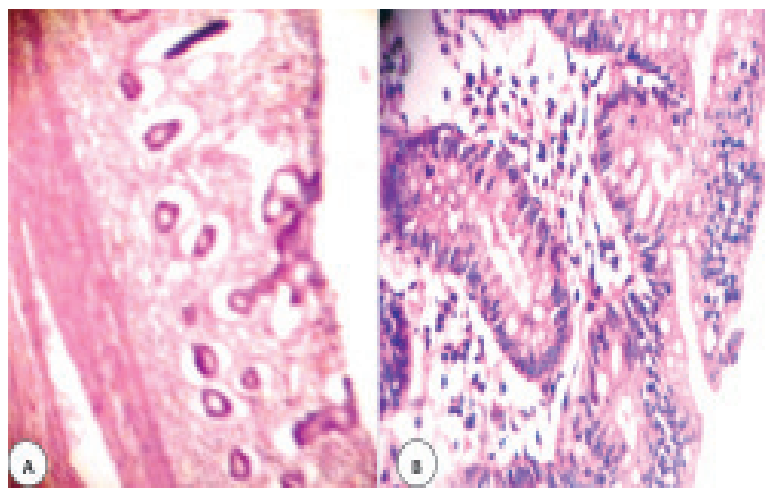

Figure 3: Microscopic picture of ileum. A. Flat mucosa and villus atrophy, marked by arrow(10x 10). B. The lamina propria showing infiltration of inflammatory cells, marked by arrow. (40x 10) 


\section{Discussion}

The word 'bezoar' derives from either the Arabic term 'badzehr' or the Persian world, padzahr' both of which denote counter poison or antidote. This word applied to a greenish, hard concretion found in the fourth stomach of the Syrian goat. The stone was felt to prevent poisoning and came to Europe as the bezoar-stone which was highly prized for its medicinal properties. ${ }^{9}$

A bezoar is the result of the ingestion of indigestible or poorly digestible substances that accumulate in the gastrointestinal tract in the form of a mass. ${ }^{10}$

Depending on the content there are different types of bezoar. These includes- phytobezoar- composed of plant material fibers; trichobezoar- composed of hair; lactobezoar - that are formed in infant from constant intake of inspissated milk; pharmacobezoar- that are formed by sustained release medication; lithobezoar formed by rock like substances or combination like trichophytobezoar. ${ }^{3}$ Cases of unusual types of bezoars like medications, shellac, cement, cotton, dry fruit and cushion foam, etc. were also on record. ${ }^{11-15}$

The stomach is the most common site of bezoar formation, followed by the small intestine and, rarely, the colon or rectum. ${ }^{16,17}$

Among all type of bezoar phytobezoars are the most common type and formed by excessive consumption of herbal nutrients containing high amounts of indigestible fibers, such as cellulose, hemicellulose, lignin and fruit tannins. ${ }^{3}$

There are some predisposing factors for gastrointestinal phytobezoars formation. These include loss of pyloric function, decreased gastric motility as well as acid secretion following gastric surgery, adhesions due to abdominal surgery, inadequate chewing, and excessive consumption of herbal nutrients including high amounts of indigestible fibers. ${ }^{18-19}$ Furthermore, delayed gastric emptying, resulting from diabetic neuropathy or hypothyroidism or connective tissue diseases also play a role in its development. ${ }^{2}$

In this case the bezoar was a phytobezoar as it was composed of undigested food fibers macroscopically and was microscopically confirmed as of vegetable or plant origin. The patient had malabsorption for many years and used to take herbal medicine which might contributed in phytobezoar formation.
The main clinical symptoms of bezoars are abdominal pain, abdominal distress, constipation, nausea and vomiting. Whereas decreased bowel sounds, rebound tenderness, rigidity and abdominal distension are found in abdominal examination. ${ }^{4}$ These can also present with complications such as intestinal obstruction or with perforation, ulcer or melena caused by mucosal irritation by the bezoars. ${ }^{5}$

Here the patient presented with abdominal pain, abdominal distress, constipation as well as features of intestinal obstruction and a palpable mass in right side of lower abdomen. Although his vital parameter was normal during admission he was found to have mild anemia on routine hematological investigations. Anemia may resulted from malabsorption as he was previously diagnosed to have chronic duodenitis with partial villous atrophy and his right hemicolectomy sample also showed chronic ileitis and atrophied villi. Both of these were histological features of malabsoption.

Small bowel obstruction is the most common major complication of phytobezoars. However, it accounts for only $0.4-4 \%$ of total cases of intestinal obstruction. ${ }^{6-}$ ${ }^{7}$ Moreover, ulcer and perforation may be seen.

The usual site of obstruction is terminal ileum and jejunum, which is the narrowest part of the small intestine. ${ }^{7}$ It can also occur as an extension of gastric phytobezoars. ${ }^{7}$ Krausz et al. found phytobezoars were most common in small intestine $(70.7 \%)$, followed by both in the small intestine and stomach $(17.6 \%)$ then in the stomach alone $(11.5 \%) .{ }^{20}$ Similar finding were also found by Chisholm et al ${ }^{12}$ and Ertugrul et al. ${ }^{3}$

The present case of phytobezoar was complicated by intestinal obstruction and terminal ileum was the site of obstruction.

Radiological imaging methods, such as plain abdominal radiography in the standing position, abdominal ultrasound and abdominal computed tomography, are important tools, used for the diagnosis of intestinal phytobezoars. Plain abdominal radiographs may show dilated intestinal loops, air-fluid levels and thickened intestinal wall. ${ }^{7}$ Ultrasonographically it may appear as an echogenic intraluminal mass having acoustic shadowing. ${ }^{22}$ On abdominal computed tomography it may show air bubbles containing intraluminal mass with dilated proximal bowl. ${ }^{7}$ 
Similarly abdominal ultrasonography of this case revealed a 7x $6 \mathrm{~cm}$ mass at ileocecal junction causing intestinal obstruction represented by excessive bowl gas.

The methods for removing bezoars in the colon can differ according to the location of obstruction, the size of the bezoar, and the presence of complications. ${ }^{1}$

Gastric lavage, and endoscopic or surgical removal, can be used in the treatment of gastric phytobezoars. Substances such as L-cysteine, metoclopramide with cellulose, papain with cellulose, pineapple juice, normal saline solution, sodium bicarbonate, hydrochloric acid, pancrelipase, pancreatin, 1-2\% zinc chloride, and coca cola can be used for the disintegration of the bezoar during gastric lavage. ${ }^{3}$

When bezoar is located in the terminal part of colon, a conservative treatment with an enema or a resin is first used. If conservative treatment fails, or in complicated cases, such as cases associated with intestinal obstruction, surgical treatment along with administration of broad-spectrum antibiotics and fluids and electrolyte correction are required. Surgical therapy may be performed either by open or laparoscopic technique. Main surgical techniques include manual fragmentation and milking into caecum, and enterotomy, Resection and anastomosis are done in complicated cases. ${ }^{3}$

In this case the patient was treated with right sided hemicolectomy under general anaesthesia as he was presented with intestinal obstruction, a complication of bezoar.

Krausz et al. reported early morbidity and mortality rates of bezoars were $11,5 \%$ and $1.7 \%$ respectively. ${ }^{20}$ Whereas no mortality and $7.6 \%$ morbidity were observed in the study conducted by Ertugrul et al. ${ }^{3}$

\section{Conclusion}

Intestinal phytobezoar is a rare clinical condition, difficult to treat and diagnose. Prevention is the best way to manage the disease. Therefore, excessive consumption of herbal nutrients, containing high amounts of indigestible fibers, should be avoided by people with a history of malabsorption.

\section{Conflict of interest: None}

\section{References}

1. Senol M, Ozdemir ZU, Sahiner IT, Ozdemir H. Intestinal obstruction due to colonic lithobezoar: A rare case report. Case Rep Pediat 2013; 2013: 854975.

2. Yoon SS, Kim MS, Kang DY, Yun TS, Jeon JH, Lee YK, et al. A case of successful colonoscopic treatment of colonic obstruction caused by phytobezoar. J Korean Soc Coloproctol 2011; 27(4): 211-14
3. Ertugrul G, Coskun M, Sevinc M, Yelimilies B, Ertugrul Fand Toydemir T. A rare cause of gastrointestinal phytobezoar: diospyros lotus.World J Emerg Sur 2012; 7: 19

4. Krausz MM, Moriel EZ, Ayalon A, Pode D, Durst AL. Surgical aspects of gastrointestinal persimmon phytobezoar treatment. Am J Surg 1986; 152: 526-30.

5. Park JW, Park JG, Kwon OK, Lee KU, Hong SC, Choe KJ et al. Clinical analysis of Bezoars. Korean J Gastroenterol 1991; 23: $32-38$.

6. Bala M, Appelbaum L,Almogy G. Unexpected cause of large bowel obstruction: colonic bezoar. Isr Med Assoc J 2008; 10 : 829-30.

7. Teo $\mathrm{M}$, Wong $\mathrm{CH}$, Chui $\mathrm{CH}$. Food bolus - an uncommon cause of small intestinal obstruction. Aust N Z J Surg 2003; 73(1): 47.

8. Kim JH, Ha HK, Sohn MJ, Kim AY, Kim TK, Kim PN. CT findings of phytobezoar associated with small bowel obstruction. Eur Radiol 2003; 13: 299-304.

9. Chisholm EM, Leong HT, Li Ak. Phytobezoar: an uncommon cause of small bowel obstruction. Ann R Coll Surg Engl 1992; 74(5): 342-44.

10. Chauhan NS, Sood D. Case report: Colonic bezoar due to Box Myrtle seeds: A very rare case report. Indian J Radiol Imaging 2011; 21(1): 21-23.

11. Andrus CH, Ponsky JL. Bezoars: classification, pathophysiology and treatment. Am J Gastroentrol 1998; 83: 476-78.

12. O’Malley JA, Ferrucci JT, Jr, Goodgame JT., Jr Medication bezoar: intestinal obstruction by an isocal bezoar.Case report and review of literature. Gastrointest Radiol 1981; 6: 141-44.

13. Chintamani, Durkhure R, Singh JP, Singhal V. Cotton Bezoar - a rare cause of intestinal obstruction: case report. BMC Surg 2003; $3: 5$.

14. Ortiz-Hidalgo C, Cuesta-Mejias T, Cervantes-Castro J. Dry fruit bezoar causing acute small intestinal obstruction. Int $\mathrm{J}$ Surg Pathol 2007; 15: 66-67.

15. Chiu B, Ciaccio C, West MA. Intestinal obstruction from furniture cushion foam bezoar. Surgery 2005; 138: 956-58.

16. Erzurumlu K, Malazgirt Z, Bektas A, Dervisoglu A, Polat C, Senyurek $\mathrm{G}$ et al. Gastrointestinal bezoars: A retrospective analysis of 34 cases. World J Gastroentrol 2005; 11: 1813-17.

17. Minty B, Kelly L. Rectal bezoars in children. CMAJ 2010; 182: 532

18. Saeed ZA, Rabassa AA, Anand BS. An endoscopic method for removal of duodenal phytobezoars. Gastrointest Endosc 1995; 41(1): 74-76.

19. Hayes PG, Rotstein OD. Gastrointestinal phytobezoars: Presentation and management. Can J Surg 1986; 29: 419-20.

20. Krausz MM, Moriel EZ, Ayalon A, Pode D, Durst AL. Surgical aspects of gastrointestinal persimmon phytobezoar treatment. Am J Surg 1986; 152: 526-30.

21. Chisholm EM, Chung SCS, Leong HT. Phytobezoar: an uncommon cause of small bowel obstruction. Ann R Coll Surg Engl 1992; 74: 342-44.

22. Ko YT, Lim JH, Lee DH, Yoon Y. Small intestinal phytobezoar: Sonographic detection. Abdom Imaging 1993; 18: 271-73. 